\title{
Highly sensitive polarization rotation measurement through a high order vector beam generated by a metasurface
}

\author{
Fuyong Yue ${ }^{1, *}$, Vincenzo Aglieri ${ }^{1,2,3}$, Roberto Macaluso ${ }^{3}$, Riccardo Piccoli ${ }^{1}$, Andrea \\ Toma $^{2}$, Luca Razzari ${ }^{1 *}$, Roberto Morandotti ${ }^{1,4,5 *}$ \\ ${ }^{1}$ INRS-EMT, 1650 Boulevard Lionel-Boulet, Varennes, Québec J3X 1S2, Canada. \\ ${ }^{2}$ Istituto Italiano di Tecnologia, Via Morego 30, 16163 Genova, Italy. \\ ${ }^{3}$ Università degli Studi di Palermo, Dipartimento di Ingegneria, Viale delle Scienze, 90128, \\ Palermo, Italy \\ ${ }^{4}$ Institute of Fundamental and Frontier Sciences, University of Electronic Science and \\ Technology of China, Chengdu 610054, Sichuan, China. \\ ${ }^{5}$ ITMO University, St. Petersburg 199034, Russia.
}

\begin{abstract}
:
The precise determination of the polarization state of light is fundamental for a vast variety of applications in remote sensing, astronomy, optics and terahertz technology, to name just a few. Typically, polarization characterization is performed by using a combination of multiple optical devices such as beam splitters, polarizers, and waveplates. Moreover, to achieve highprecision, balanced photodetectors and lock-in amplifiers are employed, thus contributing to increasing system complexity. Here, a technique for polarization rotation measurements with a dynamic range of $180^{\circ}$ and a sensitivity of about 10-2 degrees is realized using a properly designed metasurface. Such device generates a vector beam with an azimuthally-dependent polarization distribution, as a result of the superposition of two vortex beams carrying opposite orbital angular momenta $(\ell= \pm 30)$. After propagation through a linear polarizer, the spatial intensity profile of such a beam turns into 60 lobes. By tracking the displacement of only two of these lobes on a camera, the rotation of the input polarization state can be retrieved with high resolution. The proposed approach offers a new route toward the development of compact high-precision polarimeters and can also be exploited in quantum information processing, optical communications, as well as nonlinear and chiral optics.
\end{abstract}

KEYWORDS: orbital angular momentum of light; metasurface; polarization measurement; vector beams 


\section{Introduction}

Polarization is one of the fundamental properties of light and determines how the latter interacts with matter. Its measurement results therefore essential in nearly all areas of science and technology such as astronomy ${ }^{1,2}$, optics and photonics ${ }^{3,4}$, chemistry $^{5}$, liquid crystal industry ${ }^{6}$, remote sensing ${ }^{7}$, and terahertz technology $y^{8}$, to name just a few. Unlike intensity and frequency, the characterization of the polarization state of light is more challenging because of its vectorial nature. For instance, the polarization rotation of a linear polarized light beam can be retrieved by measuring the amplitude changes of its two orthogonal components. To achieve high sensitivity, a system comprising a balanced-photodetector and a lock-in amplifier has to be implemented ${ }^{3}$. However, this approach still fails to distinguish the sign of the rotation angle since the relative phase information of the two components is lost. In order to measure the rotation angle in real time, another set of polarization beam splitters and polarizers is needed, which inevitably makes the system more complex and bulky. Recently, advanced architectures based on q-plates have been proposed and allowed to achieve sensitivity of about $10^{-2}$ degrees ${ }^{9}$. However, such approach hampers the development of miniaturized and integrated devices for polarization rotation measurements.

Over the past years, "flat optics" based on metasurfaces have emerged as a promising paradigm to fully control light with ultra-thin and planar devices ${ }^{10-15}$. Such metasurfaces - arrays of scatters smaller than the free-space wavelength of light - have been the focus of an intensive research, both in the linear and nonlinear regime, due to their unique capabilities of tailoring the spatial phase and polarization of light on a subwavelength scale. Various flat metadevices including holograms ${ }^{15,16}$, lenses ${ }^{17}$ and waveplates ${ }^{18}$, as well as vector beam generators ${ }^{19}$ have been demonstrated using dielectric and plasmonic metasurfaces. Nowadays, these components constitute the cornerstones of a solid technology that can play a decisive role in systems for fluorescence imaging ${ }^{20}$, light-field imaging ${ }^{21}$, and augmented reality ${ }^{22}$, since they provide unique advantages such as the elimination of chromatic aberration, polarization selectivity, and high numerical aperture. Regarding polarization measurements, advances in metasurface technology have enabled the possibility of reducing system complexity and dimensions. Metasurface-based ellipsometry ${ }^{23}$ and polarimetry ${ }^{24-26}$ have been demonstrated as a proof-ofprinciple. However, the search for a simple and compact technique for measuring polarization rotations with high level of accuracy still challenges both academy and industry. Over the past decades, structured light beams, such as vortex and vector beams, have been widely exploited for quantum memories ${ }^{27}$, particle trapping ${ }^{28}$, high-resolution lithography ${ }^{29,30}$, as well as highspeed sensing ${ }^{31}$. Here, we demonstrate both theoretically and experimentally a simple 
metasurface-based technique, which exploits the nature of vector beams to precisely measure polarization rotations. Such vector beams (i.e., beams with "gear-like" polarization profile) arise from the superposition of two vortex beams carrying opposite spins as well as orbital angular momenta (OAM), and can be readily produced using a single reflective metasurface illuminated by a linearly polarized light beam. After propagation through a linear polarizer, the spatial intensity profile of the vector beam turns into a lobed fringe pattern (as shown in Fig. 1). The polarization rotation angle of the incident light beam can be simply retrieved by recording the rotation of these lobes with a camera. As a proof-of-principle, we employ a metasurface superimposing two OAMs with topological charge $\ell= \pm 30$ on an incident light beam to demonstrate polarization rotation measurement with a sensitivity as high as $10^{-2}$ degrees and a dynamic range of $90^{\circ}$.

\section{Experimental section}

We consider a plasmonic metasurface consisting of plasmonic nanoantennas (NAs) with an azimuthally dependent rotation angle $\alpha(\varphi)=\frac{\ell}{2} \varphi$. Such a pattern adds a $e^{i \ell \varphi}$ phase term to the wavefront of an incident circularly polarized beam while it is converted into a beam of opposite handedness ${ }^{11,32,33}$. Based on this principle, the metasurface generates a vortex beam carrying OAM with topological charge $\ell$ (or $-\ell$ ) under illumination with right-handed circularly polarized (RCP) light (or left-handed circular polarization (LCP) light), since the sign of the abrupt phase change depends on the handedness of the incident circular polarization. The case of linear polarization can be represented as the superposition of two circularly polarized beams with opposite handedness. Consequently, in this case, the metasurface generates a superposition of two vortex beams with opposite topological charges $\pm \ell$, and orthogonal circular polarization states. In turn, the superposition of two vortex beams with opposite topological charges and circular polarizations produces a vector beam with azimuthally dependent ("gear-like") polarization distribution" 9,19 . After propagation through a polarizer, the intensity profile results in $2|\ell|$ lobes due to the spatially dependent polarization distribution ${ }^{19,34}$. As the linear polarization of the incident beam is rotating by an angle $\Delta \theta$, a relative phase difference of $2 \Delta \theta$ arises between the two orthogonal circular polarization states. This phase difference is then transferred to the two generated vortex beams, thus causing the rotation of the overall polarization spatial profile of the resulting vector beam. Therefore, the lobes produced after the polarizer rotates accordingly and their positions can be simply recorded on a camera. 


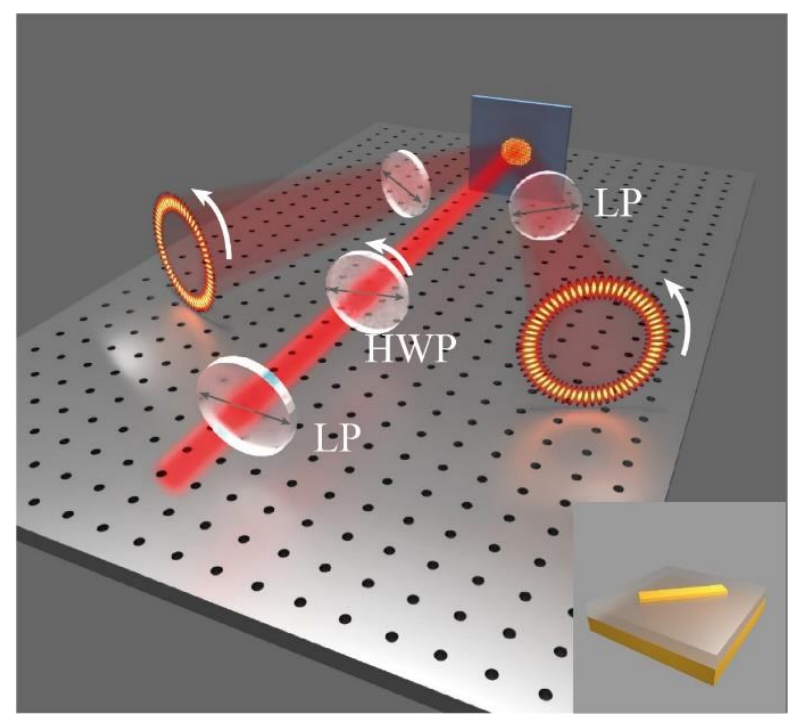

Figure 1. Schematic of setup. The polarization state of input beam is controlled by a linear polarizer (LP) and a half-wave plate (HWP). The intensity profile of the beam reflected by the metasurface has $2|\ell|$ lobes after propagation through a linear polarizer (LP). Note that two vector beams are simultaneously generated from the metasurface. The inset shows the unit cell of the metasurface composed of a gold nanoantenna (440-nm long, 80$\mathrm{nm}$ wide, and 30-nm thick) and a gold film (150-nm thick) separated by a $\mathrm{SiO}_{2}$ layer (220-nm thick). The unit cell size is $500 \mathrm{~nm}$.

We design a metasurface generating vector beams, arised from the superposition of two vortex beams carrying OAMs of $\ell= \pm 30$ to demonstrate polarization rotation measurement. Our measurement system simply consists of the reflective metasurface, a linear polarizer, and an InGaAs camera (Xenics XEVA-1781), without any additional polarization beam splitter, objectives, or wave plate. A laser (Thorlabs S1FC150) centered at $1550 \mathrm{~nm}$ is impinging on the metasurface at normal incidence and two vector beams with azimuthally dependent polarization are generated on both sides with deflection angles of $\pm 31^{\circ}$, as shown in Fig. 1 . The advantage of this off-axis design is to avoid the presence of residual unconverted light, which instead is reflected in the normal direction. The polarization state of the incident beam is controlled by a linear polarizer and a half-wave plate. The latter is mounted in a rotary stage moved by a motion controller (Newport ESP300), which is used to precisely control the linear polarization direction of the incident beam. The metasurface consists of an extremely dense array of 500,000 gold nanoantennas with identical size, but different rotation angles (on the plane). They are positioned in a square lattice with period of $500 \mathrm{~nm}$ and cover a circular area of about $400 \mu \mathrm{m}$ in diameter. A 220 -nm-thick silica $\left(\mathrm{SiO}_{2}\right)$ spacer layer and a 150 -nm-thick gold ground layer are deposited on a silicon substrate prior to nanoantenna fabrication. This reflective design, shown in the inset of Fig. 1, allow to achieve broadband operation and high conversion efficiency ${ }^{15,35}$. 
(a)

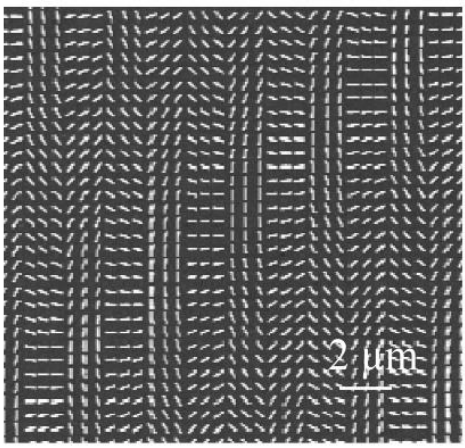

(d)

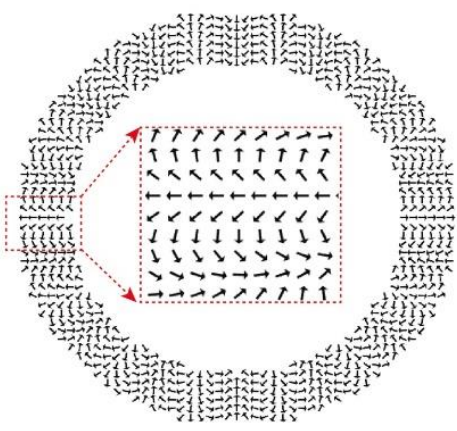

(b)

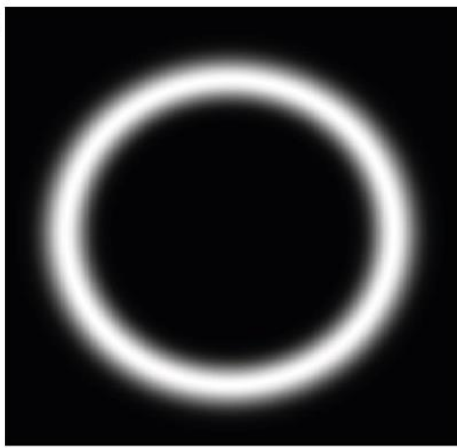

(e)

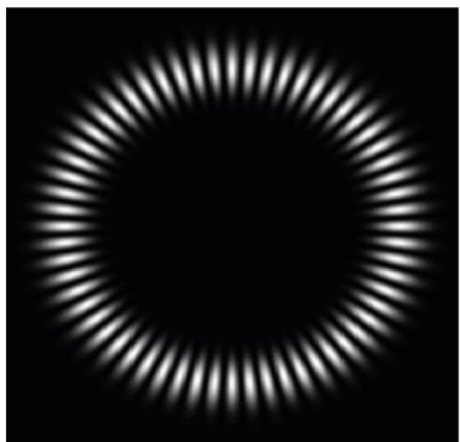

(c)

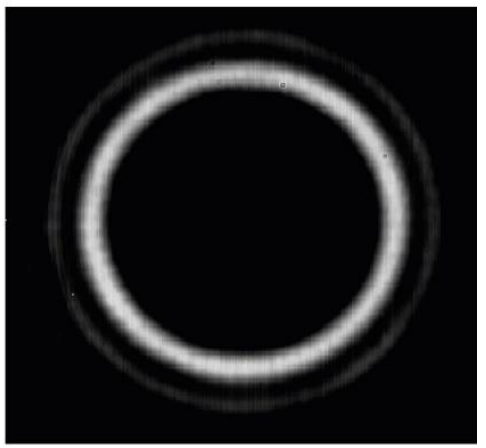

(f)

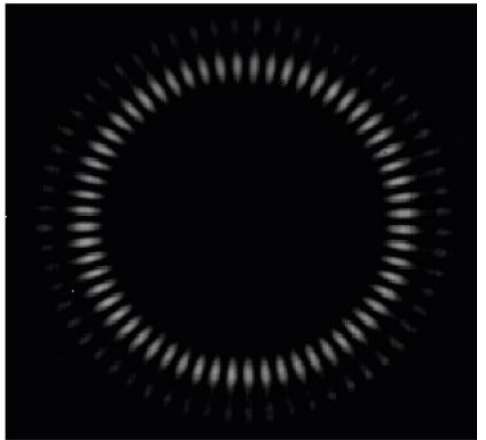

Figure 2. (a) SEM images of the fabricated metasurface sample. (b) Simulated and (c) experimental intensity profiles of the vector beam. (d) Polarization profile of the generated vector beam. (e) Simulated and (f) experimental intensity profiles after propagation through a linear polarizer.

The metasurface is fabricated by using electron-beam lithography and standard deposition techniques. Figure 2 (a) shows the scanning electron microscopy (SEM) image of the fabricated sample. The conversion efficiency of this metasurface, defined as the power of the generated beams in both sides divided by the power of the incident beam, is more than $40 \%$ over spectral range of $1300-1650 \mathrm{~nm}$. First, the metasurface is illuminated with a linearly polarized Gaussian beam to generate the desired vector beam. A lens with focal length of $50 \mathrm{~mm}$ is used, in this case, to image the entire beam profile on the camera (such lens is then removed during polarization rotation measurements, where only two lobes are recorded). The simulated and experimental intensity profiles of the vector beam are shown in Fig. 2 (b) and (c), respectively. As expected, the resulting beam features a doughnut-shaped intensity profile with a radius depending on both the beam radius $w(z)$ and its topological charge $\ell$ as $R=w(z) \sqrt{\ell / 2}$. The calculated azimuthally dependent polarization distribution is illustrated in Fig. 2 (d). After propagation through a linear polarizer, the intensity profile is expected to form an azimuthally dependent fringe pattern consisting of $2|\ell|=60$ lobes. The simulated intensity profile after the polarizer is illustrated in Fig. 2 (e) and shows a very good agreement with the 
experimentally recorded one, reported in Fig. 2 (f): the uniform shape and size of the experimentally retrieved lobes clearly indicate the high quality achieved during the fabrication process as well as the correct operation of the metasurface.
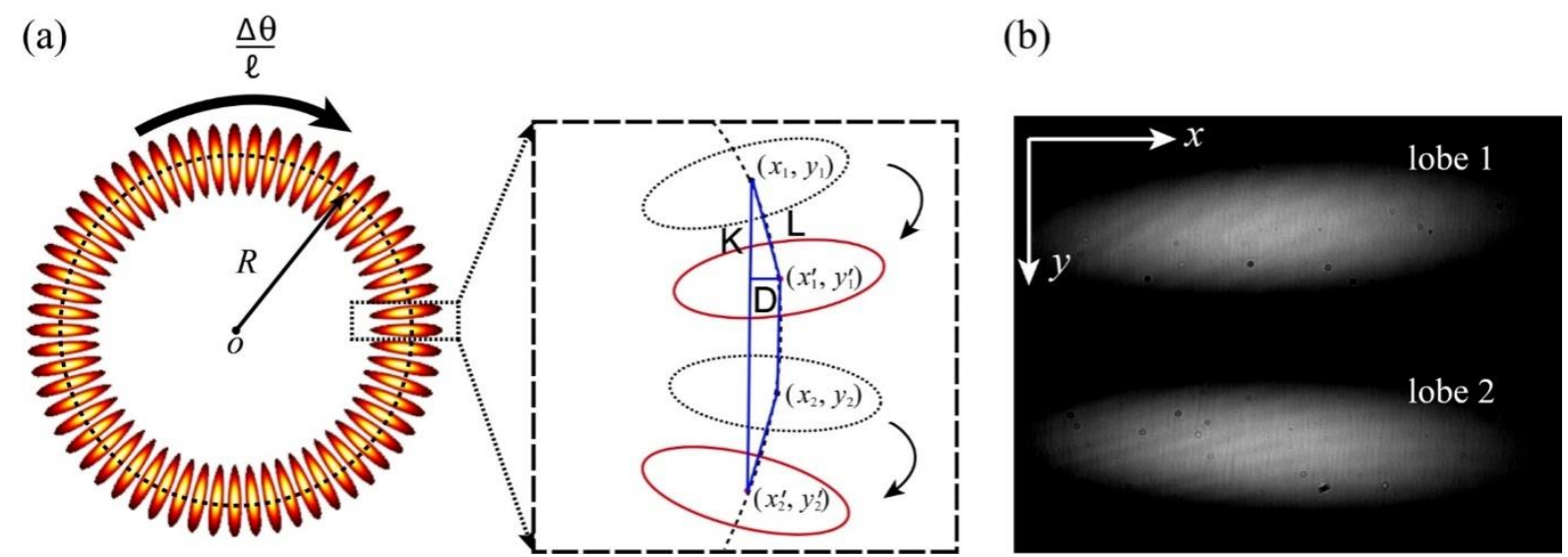

Figure 3. (a) Schematic of the operating principle of the polarization rotation measurement. (b) Image of two lobes captured by the camera.

The key point of this method is to precisely measure the rotation angle of the lobes which directly relates to the input polarization rotation angle. This can be obtained by tracking the position of the lobes on a camera, as illustrated in Fig. 3 (a). Figure 3 (b) shows the image of two lobes recorded by the InGaAs camera. The camera has $320 \times 256$ pixels with size of $30 \mu \mathrm{m} \times 30 \mu \mathrm{m}$. The intensity "centre of mass" of the lobes (which are represented by the black dashed ellipses in Fig. 3 (a)) is given in Cartesian coordinates by $x_{i}=\frac{\sum x_{i} \cdot I\left(x_{i}, y_{i}\right)}{\sum I\left(x_{i}, y_{i}\right)}, y_{i}=$ $\frac{\sum y_{i} \cdot I\left(x_{i}, y_{i}\right)}{\sum I\left(x_{i}, y_{i}\right)}$, where $I\left(x_{i}, y_{i}\right)$ is the corresponding intensity distribution. As the input polarization is rotated of an angle $\Delta \theta$, the centre of mass of the lobes rotate of $\frac{\Delta \theta}{\ell}$, to the new positions $\left(x_{i}^{\prime}, y_{i}^{\prime}\right)$ (see the red ellipses in Fig. 3 (a)). Then, the rotation angle can be easily retrieved as $\Delta \theta=2 \ell \cdot \operatorname{asin}\left(\frac{L}{2 R}\right)$, where $L$ is the length of chord connecting $\left(x_{i}, y_{i}\right)$ and $\left(x_{i}^{\prime}, y_{i}^{\prime}\right)$ and $R$ is the radius of the entire beam. The radius is easily extracted from the positions of two adjacent lobes as $R=\frac{\sqrt{\left(x_{1}-x_{2}\right)^{2}+\left(y_{1}-y_{2}\right)^{2}}}{2 \cdot \sin (\pi / 2 \ell)}$, thus not requiring the imaging of the whole beam. The chord length can be obtained from the original position and the new position of one lobe as $L=$ $\sqrt{\left(x_{i}-x_{i}^{\prime}\right)^{2}+\left(y_{i}-y_{i}^{\prime}\right)^{2}}$. We refer to this method as "one-lobe method". To verify its effectiveness, we rotate the polarization of the incident beam by $9^{\circ}, 1^{\circ}$, and $0.2^{\circ}$, and retrieve the values with the above-reported equations. Each measurement is repeated 50 times. To increase the signal-to-noise ratio, the intensity centre of mass of the lobes is calculated by 
averaging 20 frames. Table 1 ("one-lobe" column) shows the calculated best estimates (averaging the 50 values), standard deviations, and relative errors (i.e., the difference between the best estimates and the actual value divided by the actual value itself). As it is possible to see, for the case of $9^{\circ}$ and $1^{\circ}$, the rotation angle is successfully retrieved with acceptable relative errors using the "one-lobe method". However, this method produces significant error for small rotation angles, such as the case of $0.2^{\circ}$. In order to enhance the accuracy, we introduce the "two-lobe method", where two adjacent lobes are simultaneously used. Here, the chord length is calculated from the original and new positions of two adjacent lobes as $L=$ $\sqrt{K^{2}+D^{2}} \quad, \quad$ where $\quad K=\frac{\sqrt{\left(x_{2}^{\prime}-x_{1}\right)^{2}+\left(y_{2}^{\prime}-y_{1}\right)^{2}}-\sqrt{\left(x_{1}^{\prime}-x_{2}\right)^{2}+\left(y_{1}^{\prime}-y_{2}\right)^{2}}}{2}$ and $D=$ $\sqrt{\left[\frac{\left(x_{1}+x_{2}^{\prime}\right)}{2}-\frac{\left(x_{2}+x_{1}^{\prime}\right)}{2}\right]^{2}+\left[\frac{\left(y_{1}+y_{2}^{\prime}\right)}{2}-\frac{\left(y_{2}+y_{1}^{\prime}\right)}{2}\right]^{2}}$ (see schematic in Fig. 3 (a)). $K$ can assume positive or negative values, which correspond to clockwise and anticlockwise rotations, respectively. The calculated data using the two-lobe method is shown in Table 1 ("two-lobe" column). As it can be observed, the two-lobe method is more robust and accurate compared to the one-lobe method, especially in the case of very little rotation angles. Remarkably, for the rotation angle of $0.2^{\circ}$, the two-lobe method yields very high accuracy with a relative error of only $-3 \%$.

Table 1. The calculated values of best estimates, standard deviations, and relative errors based on the one-lobe and two-lobe methods.

\begin{tabular}{c|c|c|c|c|c|c} 
Rotation angle & \multicolumn{2}{|c|}{ Best estimate } & \multicolumn{2}{c|}{ Standard deviation } & \multicolumn{2}{c}{ Relative error } \\
\hline & One-lobe & Two-lobe & One-lobe & Two-lobe & One-lobe & Two-lobe \\
$9^{\circ}$ & $9.836^{\circ}$ & $9.075^{\circ}$ & 0.18 & 0.016 & $9.3 \%$ & $0.8 \%$ \\
$1^{\circ}$ & $1.032^{\circ}$ & $0.951^{\circ}$ & 0.06 & 0.01 & $3.2 \%$ & $-4.9 \%$ \\
$0.2^{\circ}$ & $0.433^{\circ}$ & $0.194^{\circ}$ & 0.21 & 0.017 & $116 \%$ & $-3 \%$ \\
\hline
\end{tabular}

To further prove the robustness and high sensitivity of our two-lobe method, we try different angle steps and dynamic ranges. In this case, the centre of the lobes is calculated after averaging over 200 frames to reduce random noise. As it can be seen in Fig. 4, the experimentally retrieved values closely follow the actual polarization rotation angles of the incident laser beam. Even for very small step angle of $0.04^{\circ}$, the measured values show good agreement with the actual ones. The error bars in Fig. 4 account for power fluctuations of the laser source, mechanical vibrations, and fabrication imperfections. When rotating the polarization angle of $180^{\circ}$, a lobe is completely replaced by an adjacent one. The recorded lobe motion as a function of the input polarization rotation is available in a supplementary video. 


\section{Conclusion}

In conclusion, we have introduced a metasurface-based technique exploiting the OAM of light to precisely measure polarization rotations. As a proof-of-principle, we have demonstrated the capability of sensing rotation angles in the range of $-45^{\circ}$ to $45^{\circ}$ with a sensitivity of $\sim 10^{-2}$ degrees, using a vector beam arising from the superposition of two vortex beams carrying OAM of $\ell= \pm 30$. In our method, a metasurface transforms the linear polarization state of an incident light beam into an azimuthally dependent polarization distribution. Then, a linear polarizer turns the vector beam into an intensity profile featuring $2|\ell|$ lobes, which rotate according to the polarization rotation angle of the input beam. This rotation can be retrieved by simply tracking the position of two adjacent lobes on a camera. This approach has the advantage of precisely measuring the rotation angle of polarization and its sign, without the need of bulky optical components and sophisticated electronic devices. The footprint of the system can be reduced, towards integration, by manufacturing the metasurface and the polarizer on a single substrate. Moreover, as the order of the OAM rises, the number of lobes increases accordingly. Their shapes become more elongated and their displacements, as a function of the polarization rotation angle, result smaller. Therefore, the chord length $L$ can be replaced by the vertical shift $K$ since $D$ becomes negligible. In this scenario, the measurement can be further simplified and the camera can be substituted by a photodetector array, which simply records the vertical shift $K$. While our metasurface was designed to work in the telecom band, which is a significant region for practical implementations, it is worth mentioning that the proposed approach can be extended to other wavelengths of interests ranging from the visible to the $\mathrm{THz}^{37-41}$. In addition, we have successfully demonstrated a reflective metasurface capable of efficiently generating a superposition of OAM states with extremely large $\ell$ values and orthogonal circular polarizations that - beyond this investigation - can find interesting applications in OAM-based communications ${ }^{42}$, high-order Pancharatnam-Berry phase measurements ${ }^{34}$, and quantum information processing ${ }^{43}$. 
(a)

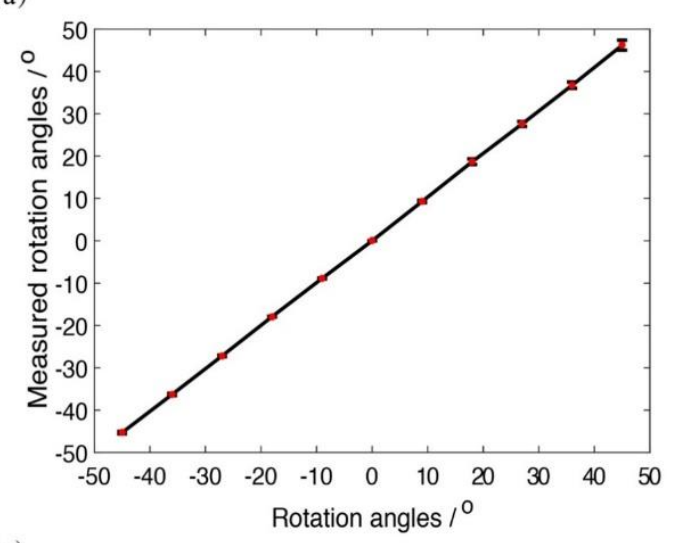

(c)

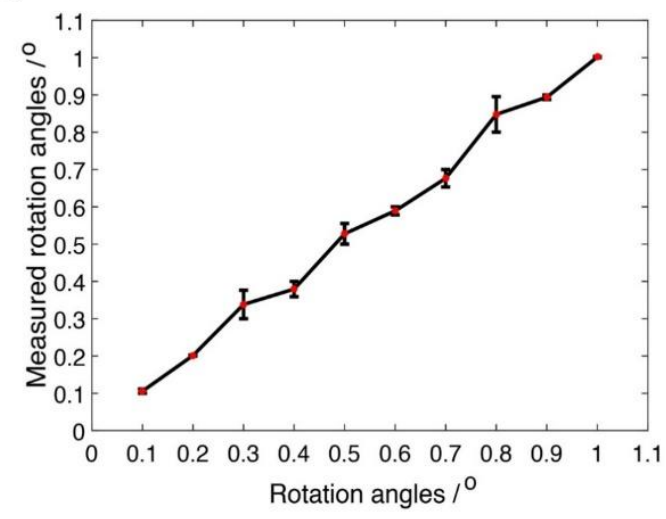

(b)

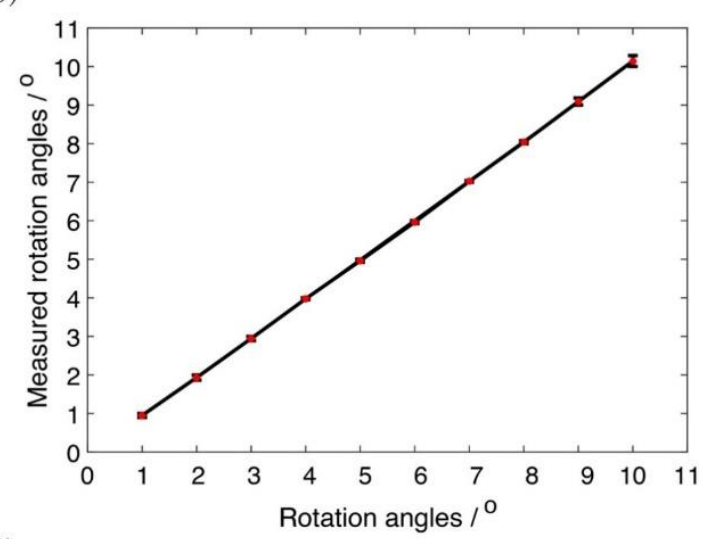

(d)

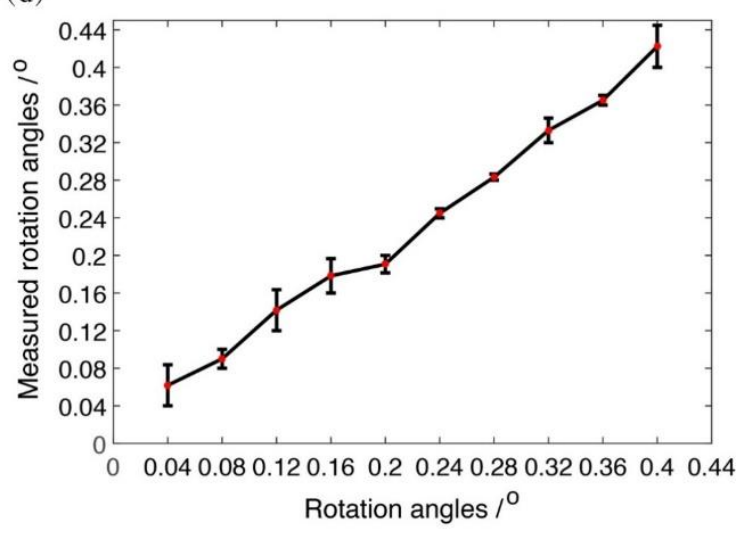

Figure 4. Measured rotation angles within different ranges and with different angle steps: (a) range from $-45^{\circ}$ to $45^{\circ}$ with step of $9^{\circ}$; (b) range from $1^{\circ}$ to $10^{\circ}$ with step of $1^{\circ}$; (c) range from $0.1^{\circ}$ to $1^{\circ}$ with step of $0.1^{\circ}$; (d) range from $0.04^{\circ}$ to $0.44^{\circ}$ with step of $0.04^{\circ}$.

\section{AUTHOR INFORMATION}

Corresponding author

*Email: fuyong.yue@emt.inrs.ca

*Email: razzari@emt.inrs.ca

*Email: morandotti@emt.inrs.ca

\section{ACKNOWLEDGEMENT}

This work at INRS was supported by the Natural Sciences and Engineering Research Council of Canada (NSERC) through the Strategic and Discovery grant programs as well as by the Canada Research Chair Program. R.Mo. acknowledges additional support from the Government of the Russian Federation through the ITMO Fellowship and Professorship Program (Grant No. 074-U 01) and from the 1000 Talents Sichuan Program in China. 


\section{REFERENCES}

1 Lei, F., Dean, A. \& Hills, G. Compton polarimetry in gamma-ray astronomy. Space Science Reviews82, 309-388 (1997).

2 Tinbergen, J. Astronomical polarimetry. (Cambridge University Press, 2005).

3 Chang, C.-Y., Wang, L., Shy, J.-T., Lin, C.-E. \& Chou, C. Sensitive Faraday rotation measurement with auto-balanced photodetection. Review of Scientific Instruments82, 063112 (2011).

4 Dey, P., Shukla, R. \& Venkateswarlu, D. High magnetic field measurement utilizing Faraday rotation in SF11 glass in simplified diagnostics. Applied Optics56, 2873, doi:10.1364/AO.56.002873 (2017).

5 Bobbitt, D. R. \& Yeung, E. S. Direct and indirect polarimetry for detection in microbore liquid chromatography. Analytical Chemistry56, 1577-1581 (1984).

6 Wu, S.-T., Efron, U. \& Hess, L. D. Birefringence measurements of liquid crystals. Applied optics 23, 3911-3915 (1984).

7 Tyo, J. S., Goldstein, D. L., Chenault, D. B. \& Shaw, J. A. Review of passive imaging polarimetry for remote sensing applications. Applied optics45, 5453-5469 (2006).

8 Wu, Q. \& Zhang, X. C. Ultrafast electro-optic field sensors. Applied physics letters68, 1604-1606 (1996).

9 D'ambrosio, V. et al. Photonic polarization gears for ultra-sensitive angular measurements. Nature communications4, 2432 (2013).

10 Hsiao, H.-H., Chu, C. H. \& Tsai, D. P. Fundamentals and Applications of Metasurfaces. Small Methods1, 1600064, doi:10.1002/smtd.201600064 (2017).

11 Huang, L. et al. Dispersionless phase discontinuities for controlling light propagation. Nano letters 12, 5750-5755 (2012).

12 Lin, D., Fan, P., Hasman, E. \& Brongersma, M. L. Dielectric gradient metasurface optical elements. science345, 298-302 (2014).

13 Ni, X., Kildishev, A. V. \& Shalaev, V. M. Metasurface holograms for visible light. Nature communications4, 2807 (2013).

$14 \mathrm{Yu}, \mathrm{N}$. et al. Light propagation with phase discontinuities: generalized laws of reflection and refraction. science334, 333-337 (2011).

15 Zheng, G. et al. Metasurface holograms reaching $80 \%$ efficiency. Nature nanotechnology10, 308 (2015).

16 Wen, D. et al. Helicity multiplexed broadband metasurface holograms. Nature communications6, 8241 (2015).

17 Khorasaninejad, M. et al. Metalenses at visible wavelengths: Diffraction-limited focusing and subwavelength resolution imaging. Science352, 1190-1194 (2016).

18 Kruk, S. et al. Invited Article: Broadband highly efficient dielectric metadevices for polarization control. Apl Photonics1, 030801 (2016).

19 Yue, F. et al. Multichannel Polarization-Controllable Superpositions of Orbital Angular Momentum States. Advanced Materials29, 1603838 (2017).

20 Jang, M. et al. Wavefront shaping with disorder-engineered metasurfaces. Nature photonics 12, 84 (2018).

21 Lin, R. J. et al. Achromatic metalens array for full-colour light-field imaging. Nature nanotechnology, 1 (2019).

22 Lee, G.-Y. et al. Metasurface eyepiece for augmented reality. Nature communications 9 , 4562 (2018).

23 Wen, D. et al. Metasurface for characterization of the polarization state of light. Optics express 23, 10272-10281 (2015).

24 Pors, A., Nielsen, M. G. \& Bozhevolnyi, S. I. Plasmonic metagratings for simultaneous determination of Stokes parameters. Optica2, 716-723 (2015). 
25 Espinosa-Soria, A., Rodríguez-Fortuño, F. J., Griol, A. \& Martínez, A. On-chip optimal Stokes nanopolarimetry based on spin-orbit interaction of light. Nano letters17, 31393144 (2017).

26 Ding, F., Chen, Y. \& Bozhevolnyi, S. Metasurface-based polarimeters. Applied Sciences8, 594 (2018).

27 Parigi, V. et al. Storage and retrieval of vector beams of light in a multiple-degree-offreedom quantum memory. Nature communications6, 7706 (2015).

$28 \mathrm{Ng}$, J., Lin, Z. \& Chan, C. Theory of optical trapping by an optical vortex beam. Physical review letters104, 103601 (2010).

29 Dorn, R., Quabis, S. \& Leuchs, G. Sharper focus for a radially polarized light beam. Physical review letters91, 233901 (2003).

30 Hao, X., Kuang, C., Wang, T. \& Liu, X. Phase encoding for sharper focus of the azimuthally polarized beam. Optics Letters35, 3928-3930 (2010).

31 Berg-Johansen, S. et al. Classically entangled optical beams for high-speed kinematic sensing. Optica2, 864-868 (2015).

32 Bomzon, Z. e., Biener, G., Kleiner, V. \& Hasman, E. Space-variant PancharatnamBerry phase optical elements with computer-generated subwavelength gratings. Optics letters27, 1141-1143 (2002).

33 Karimi, E. et al. Generating optical orbital angular momentum at visible wavelengths using a plasmonic metasurface. Light: Science \& Applications3, e167 (2014).

34 Milione, G., Evans, S., Nolan, D. \& Alfano, R. Higher order Pancharatnam-Berry phase and the angular momentum of light. Physical review letters108, 190401 (2012).

35 Jiang, S.-C. et al. Controlling the polarization state of light with a dispersion-free metastructure. Physical Review X4, 021026 (2014).

36 Baumann, S., Kalb, D., MacMillan, L. \& Galvez, E. Propagation dynamics of optical vortices due to Gouy phase. Optics Express 17, 9818-9827 (2009).

37 Arbabi, A., Horie, Y., Bagheri, M. \& Faraon, A. Dielectric metasurfaces for complete control of phase and polarization with subwavelength spatial resolution and high transmission. Nature nanotechnology10, 937 (2015).

38 Liu, L. et al. Broadband metasurfaces with simultaneous control of phase and amplitude. Advanced materials 26, 5031-5036 (2014).

39 Zhang, L. et al. Ultra-thin high-efficiency mid-infrared transmissive Huygens metaoptics. Nature communications9, 1481 (2018).

40 Devlin, R. C., Khorasaninejad, M., Chen, W. T., Oh, J. \& Capasso, F. Broadband highefficiency dielectric metasurfaces for the visible spectrum. Proceedings of the National Academy of Sciences113, 10473-10478 (2016).

41 Gao, L.-H. et al. Broadband diffusion of terahertz waves by multi-bit coding metasurfaces. Light: Science \& Applications4, e324 (2015).

42 Wang, J. et al. Terabit free-space data transmission employing orbital angular momentum multiplexing. Nature photonics6, 488 (2012).

43 Sit, A. et al. High-dimensional intracity quantum cryptography with structured photons. Optica4, 1006-1010 (2017). 